\title{
Optimum Position of Acoustic Emission Sensors for Ship Hull Structural Health Monitoring Based on Deep Machine Learning
}

\author{
[Vassilios Kappatos ${ }^{1}$, Petros Karvelis ${ }^{2}$, George Georgoulas ${ }^{3}$, Vasilis Tzitzilonis ${ }^{4}$ ]
}

\begin{abstract}
In this paper a method for the estimation of the optimum sensor positions for acoustic emission localization on ship hull structures is presented. The optimum sensor positions are treated as a classification (localization) problem based on a deep learning paradigm. In order to avoid complex and timeconsuming implementations, the proposed approach uses a simple feature extraction module, which significantly reduces the extremely high dimensionality of the raw signals/data. The optimum sensor position is defined by the maximum localization rate. In simulation experiments, where a stiffened plate model was partially sunk into the water, the localization rate of acoustic emission events in a noise-free environment is greater than $\mathbf{9 9 . 5}$ $\%$, using only a single sensor.
\end{abstract}

Keywords-Acoustic emission, optimum sensor positions, ship hull, deep machine learning

\section{Introduction}

Acoustic emission (AE) has gained increasing credibility in recent years [1]. AE only needs the signals from one or more relatively small sensors, attached to a structure or specimen. These sensors mainly are piezoelectric sensors and are operating in the region of $\mathrm{kHz}$ or $\mathrm{MHz}$. The structure or specimen can be exanimated in-service or in-laboratory operation while the AE system is continuously monitoring the progressive damage $[1,2]$. The damage mechanisms within the structure release small amounts of energy in the form of ultrasound, this travels through the structure and can be detected by AE sensors. This amount of energy could be crack initiation, crack growth, crack opening, crack closure, dislocation movement, phase transformation in monolithic materials, fiber breakage, fiber-matrix debonding in composites etc.

A key strength of the $\mathrm{AE}$ technique lies in the ability to locate sources of damage. At first the problem appears trivial: Using the known positions of a set of sensors, and the differences in the time-of-arrival (DTA) between the sensors, calculate the intersection of a series of hyperbolae about the transducers. The location of a signal source using DTA data is

1. Department of Technology and Innovation (ITI), University of Southern Denmark (SDU), Campusvej 55, DK-5230 Odense M, Denmark,

2. Laboratory of Knowledge and Intelligent Computing, Department of Computer Engineering, Technological Educational Institute of Epirus, Arta, Greece

3. Control Engineering Group, Department of Computer Science, Electrical and Space Engineering, University of Technology, SE-97187 Luleå, Sweden

4. Department of Electrical Engineering and Computer Technology, University of Patras, Campus, 26500, Patras, Greece a wide applicable problem such as target detection for radar, sonar and seismic signals [3]. Current $\mathrm{AE}$ source location methods, using DTA [1,4] and single sensor modal analysis location (SSMAL) [5] require a large number of sensors and careful planning to accurately locate a source. Significant complications arise due to (a) the dispersive, modal nature of ultrasound in most real structures, (b) homogeneities in the wave propagation medium and (c) the presence of noisy environment. The modal nature of the waves is countered by ensuring that the fastest mode is used at all times, but the homogeneities and complexities in the structure are less simple to overcome. Complex geometric structures make theoretical wavepath analysis difficult and time consuming. Additionally, in practice, various types of noise distort the DTA measurements, introducing differences between the estimated and the real source position. The number and the positions of sensors significantly affect the accuracy of $\mathrm{AE}$ source location $[6,7]$.

One of the most important, complex and large offshore structures is the ship hull, of which structural members must properly monitoring and rectified in order to avoid catastrophic failure with many losses of human lives and unanticipated out-of-service time. Ship hull structures suffer from several kinds of damages, including corrosion, cracking, buckling, indent etc. Corrosion and fatigue cracking are the most pervasive types of structural problems experienced in ship structures [8]. The damage modes, if not properly monitored and rectified, can potentially lead to catastrophic failure or unanticipated out-of-service time. The most corrosive areas are both the bottom plating and the side shell plating since in these areas the steel is always in contact with the corrosive salt water. The fatigue cracks observed in the areas with high stress concentrations, such as at the connection between the longitudinal and the heavy transverse members of the side shell [9]. The ship hull structures can be monitored by AE techniques [10-14] during the application of an external stress. The external dynamic stress introduced by the seawaves and cargo movements in the outside and in the inside of shell respectively is an excellent source of AE phenomena. In order to achieve better performance of the localization system, the optimum position of sensors need to be defined.

One of the ways to tackle the definition problem of the optimum AE sensor positions is to treat this as a typical classification problem. In the current work, this definition problem is approached, using a deep neural network using autoenconders for fast pretraining and different number of input vectors as well as a combination of all sensor measurements. To the best of our knowledge, this is the first application of a deep learning paradigm in this field. 


\section{Classification and background theory}

The standard way to build a classifier, given a signal (usually also referred as a time series in the computer science literature), is to extract useful and robust features which represent the original series in a relative low dimensional space. In this way, the quite high dimensionality of the signals is reduced. Then, the features are fed into a classifier (statistical, neural network, rule based etc.), which has already been train using a representative set of historic data.

\section{A. Discrete Cosine Transform}

Discrete cosine transform (DCT) is usually used for compression purposes [15]. However, it can also be used for feature extraction, transforming the original time domain signal into DCT coefficients. There are many DCT implementations. One of the most common and popular is the following $[15,16]$ :

Given a discrete signal $x[n]$ of length $N$, let $s[n]$ be

$$
s[n]=\left\{\begin{array}{cc}
x[n], & 0 \leq n \leq N-1 \\
x[2 N-n-1], & N \leq n \leq 2 N-1
\end{array}\right.
$$

then the Discrete Fourier Transform of $s[n]$ is

$$
S[k]=\sum_{n=0}^{2 N-1} s[k] \cdot W_{2 N}^{n k}, \quad 0 \leq k \leq 2 N-1
$$

and the DCT of $x[n]$ is given by

$$
V[k]=W_{2 N}^{k / 2} V[k], \quad 0 \leq k \leq N-1
$$

where $W_{N}=e^{-j 2 \pi / N}$.

The DCT creates a sequence of coefficients that have the same length as the original signal. Usually, for compression purposes, only a fraction of them (the ones with the highest magnitude) is retained. In this work, a different path was followed, applying Piecewise Aggregate Approximation (PAA) to come up with a reduced representation instead of the standard approach of retaining only part of the DCT coefficients and setting the rest equal to zero.

\section{B. Piecewise Aggregate Approximation}

PAA, was independently introduced by Error! Reference source not found., and by Error! Reference source not found. Given a discrete signal $x[n]$ of length $N$ (or a time series - since both terms were used in the literature) PAA creates a representation of length $w$,

$$
\begin{gathered}
x_{P A A}=[\bar{x}[1], \bar{x}[2], \ldots, \bar{x}[w]] \text { with: } \\
\bar{x}[i]=\frac{w}{N} \sum_{j=\frac{N}{w}(i-1)+1}^{\frac{N}{w} i} x[j], \quad i=1,2, \ldots, w
\end{gathered}
$$

Practically speaking, PAA, divides the series into equally sized windows taking the mean value of the data falling within a frame. If is not divided exactly by $w$ a slightly modified version can be applied [19, 20].

\section{Deep Neural Networks}

Deep neural networks have drawn much attention lately, and have been successfully applied in numerous applications and competitions [21]. On the other hand very few applications can be found in the available literature in the field of condition monitoring. In [22] and [23], Deep Belief Networks $(\mathrm{DBN}) \mathrm{s}$ were used for the diagnosis of faults in power transformers, and reciprocating compressor valves respectively.

One of the problems of DNN is that training can be time consuming. To alleviate this, a pretraining approach was proposed, which involved pre-training the weights of each layer as the weights of an autoencoder Error! Reference source not found. An autoencoder is a neural network that learns to map the input to an output which is a copy of the input. The weights of a series of autoencoders are stuck together to form the DNN.

The whole procedure consists of a pretraining step which involves the training of a sequence of shallow autoencoders, greedily one layer at a time, using unsupervised data and two (supervised) trainings steps: a) training of the last layer of the DNN using labeled data, and b) use of backpropagation to fine-tune the entire network (end to end) again using labeled data. This sequential training allows quite deep architectures not only to be trained within reasonable amount of time but can also lead to increased performance.

\section{Experimental setup and procedure}

A stiffened plate was used to model the side shell of a ship structure. The dimensions of the stiffened plate model (SPM) are: i) side shell $56 \times 56 \times 1 \mathrm{~cm}$, ii) longitudinal a) $56 \times 1 \times 15 \mathrm{~cm} \mathrm{~b}$ ) $56 \times 10 \times 1 \mathrm{~cm}$, iii) heavy transverse member a) web $1 \times 56 \times 37 \mathrm{~cm}$, b) face $20 \times 56 \times 1 \mathrm{~cm}$.

The outside side shell is dyed with oil paint in order to simulate as much as possible the outer surface of a real ship's side. Reflections at the end of the SPM were reduced by wrapping the ends in putty. In order to investigate the influence of water, the SPM and its supports were fixed in a water tank. The putty at the edges of the SPM prevents the passage of the water on the inside shell of the plate. The putty is dyed with oil paint for better water-tightness and to avoid putty corrosion. The fixed boundary condition of the model was obtained by clamping the side shell to three heavy bases. 
Insulation material was placed between the SPM and the three

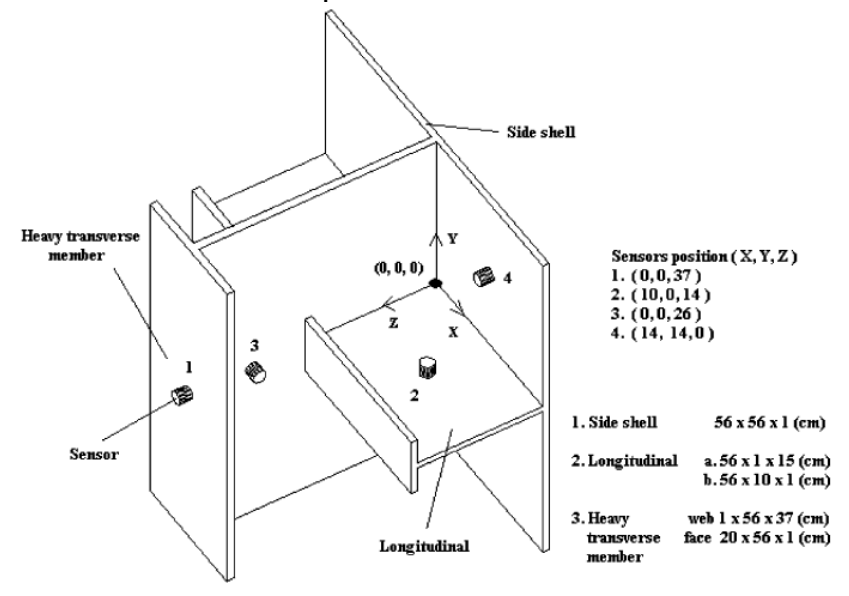

Figure 1. The stiffened plate and the sensor positions.

bases, the three bases and the bottom of the tank, at the bottom of the tank and on the floor to eliminate external noise.

Physical Acoustics Corporation (PAC) R15-Alpha sensors were used to detect waves in the steel stiffened plate. The sensors were stuck on the plate with grease couplant. In real applications, where installation cost is a significant factor, the number of sensors must be reduced and, simultaneously, the sensors must be configured at maximum sensitivity to cover the greatest possible ship hull area. In our experiments, the pulser amplitude was decreased, and the gain was set within the specification limits of the amplifiers used. This setting was close to a real-life application, where the received signal is propagated through several adjacent stiffened plates and the signal reflection at the plate edges is minimized. The sensor signals were amplified at $58 \mathrm{~dB}$ (two sensors), 70.7 and $80 \mathrm{~dB}$, digitized at $1 \mathrm{MHz}, 16$ bit accuracy and stored in the $\mathrm{AE}$ analysis system PAC Mistras 2001. The fourth channel's AE system triggers, when the signal on any channel exceeded a pre-defined threshold, and the four channel simultaneous recordings, were stored on a hard disk. The AE source was simulated with a piezoelectric pulse-generator, which is in common use [25].

AE source was simulated with a Piezoelectric PulseGenerator and in each location ten repeated measurements were recorded. Three locations/classes were simulated: (i) AEsource in the welding seam between the longitudinal and the heavy transverse member (web), (ii) AE-source in the welding seam between the longitudinal and the side shell, (iii) AEsource in the welding seam between the heavy transverse member (web) and side shell. The collected signals, each one 30720 samples long, from four different AE sensors were used for the evaluation of the proposed method. A detailed description of the experimental set up can be found in [13].

\section{Iv. Optimum sensor positions procedure on ship hull structure}

In this paper a method for the estimation of the optimum sensor positions for accurate AE localization on ship hull structures is presented. The estimation AE sensor is treated as a classification problem. Firstly, the AE signals are transformed, using the DCT, followed by the dimensionality reduction stage using PAA. After, a DNN is employed for the classification module. The optimum $\mathrm{AE}$ sensor position is defined at the maximum location rate.

Ten AE signals were acquired from 15 different locations for each location/class [13] as close as possible to the welding seam, resulting in a total of $3 \times 15 \times 10=450$ signals for each one of the four sensors. In each scenario the 10 fold cross validation procedure was employed [26].

As in [14], a feature vector of dimension 200 was created after the consecutive application of DCT and PAA. In this work however, the classification performance of the proposed scheme was evaluated using all 200 features, the first 100 features and the first 50 features (an inspection of the values for the Area Under the Receiver Operating Characteristic Curve (AUC) [27], revealed a decreasing trend of the utility of the features).

As in [14] using the rule of thumb that smaller layers should be used as we move from the input to the output, to force the neural network to generalize rather than overfit [28] three different architectures were employed depending on the size of the input vector: $200-50-10-3,100-50-10-3$ and 50-5010-3.

As in our previous work [14], sensor no 2 yields (Table I) very poor results due to the confusion of two out of three classes. This is probably due to its almost equidistance placement from the corresponding seams. Sensor 4 (Table I and Table II) achieves very high classification accuracy indicating that with the strategic placement of a sensor almost all classes can be successfully covered.

\section{v. Conclusions}

This article presented the potential use of DNNs for localization of $\mathrm{AE}$ events on the extreme complex ship hull structures. To the best of our knowledge, this is the first application of a deep learning paradigm in this field. Four different sensors where used in the experimental set-up. AE signals were acquired from different locations all of them as close as possible to the welding seam. The result was to acquire 450 signals for each one of the four sensors. Signals, first, are passing from a transformation stage using DCT, followed by the dimensionality reduction stage using PAA. After, a DNN is employed for the classification module. The localization rates achieved where, greater than $99.56 \%$, using only a single sensor. 
Proc. of the Sixth International Conference on Advances in Mechanical and Robotics Engineering - AMRE 2017.

Copyright $\odot$ Institute of Research Engineers and Doctors. All rights reserved.

ISBN: 978-1-63248-140-5 doi: 10.15224/ 978-1-63248-140-5-43

TABle I. Classification Performance.

\begin{tabular}{|c|c|c|c|}
\hline & \multicolumn{3}{|c|}{ Features } \\
\hline & $\mathbf{2 0 0}$ & $\mathbf{1 0 0}$ & $\mathbf{5 0}$ \\
\hline Sensor 1 & 93.33 & 94.89 & 92.44 \\
\hline Sensor 2 & 64.44 & 64.89 & 66.00 \\
\hline Sensor 3 & 94.67 & 97.33 & 96.89 \\
\hline Sensor 4 & 95.56 & 98.00 & $\mathbf{9 9 . 5 6}$ \\
\hline
\end{tabular}

TABLE II. AgGREGATE CONFUSION MATRIX FOR SENSOR 4, ACCURACY = $99.56 \%$.

\begin{tabular}{|c|c|c|c|c|}
\hline & & \multicolumn{3}{|c|}{ Predicted Class } \\
\hline & & $\boldsymbol{A}$ & $\boldsymbol{B}$ & $\boldsymbol{C}$ \\
\hline \multirow{3}{*}{$\begin{array}{c}\text { TRUE } \\
\text { Class }\end{array}$} & $\boldsymbol{A}$ & 150 & 0 & 0 \\
\cline { 2 - 5 } & $\boldsymbol{B}$ & 1 & 149 & 0 \\
\cline { 2 - 5 } & $\boldsymbol{C}$ & 1 & 0 & 149 \\
\hline
\end{tabular}

\section{References}

[1] R. K. Miller, E.V.K. Hill, and P.O. Moore, Nondestrucive Testing Handbook, 3rd ed, vol. 6. OH: American Society for Nondesctructive, 2005.

[2] D. O. Harris and H. L. Dunegan, "Continuous monitoring of fatigue crack growth by acoustic emission," Exper. Mech., pp. 71-81, Feb. 1974.

[3] F Gustafson, and F Gunnarsson, "Positioning using time-difference of arrival measurements", IEEE Int. Conf. Acoust. Speech Signal Process. 2003, (ICASSP). China, vol. 6, pp 553-556, Apr. 2003.

[4] H. J. Rindorf, Acoustic Emmision Source Location in Theory and in Practise, Bruel and Kjaer Technical Review 2, pp. 3-44, 1981.

[5] R. Pullin, Structural Integrity Monitoring of Steel Bridges using Acoustic Emission Techniques, Ph.D Thesis, Div. Mech. Eng. and Energy Stud., University of Wales, UK, 2001.

[6] V Kappatos, E Lympertos, and E. Dermatas. "Estimation of the best sensors position for minimum error location of acoustic emission sources on spherical surface", 3rd Int. Conf. Non-Destructive Testing, Hellenic Society for NDT, Chania, Crete, Greece, pp. 123-128, Oct. 2003.

[7] E. Lybertos, V. Kappatos, and E. Dermatas, "Comparison of acousticemission source location methods", 1st Int. Conf. Scient. Computing to Computational Eng. (IC-SCCE), Greece, Sept. 2004.

[8] U.O. Akpan, T. S. Koko, B. Ayyub, and T.E. Dunbar, "Risk assessment of aging ship hull structures in the presence of corrosion and fatigue", vol 15 (3), pp. 211-231, May-June 2002.

[9] M. R. Andersen, Fatigue Crack Initiation and Growth in Ship Structures, Ph.D. Thesis, Dept. Naval Arch. Offshore Eng. TU Denmark,. Jan. 1998.

[10] V. Kappatos, and E. Dermatas, Acoustic Emission Testing for the Monitoring and Detection of Damage to Ship Hull Structures. Theory and Uses of Acoustic Emissions, Nova Publishers, 2011.

[11] V Kappatos. G Georgoulas, C Stylios, and E. Dermatas, "Evolutionary dimensionality reduction for crack localization in ship structures using a hybrid computational intelligent approach", IEEE Int. Conf. Neural Netw. GA, pp 1531-1538, Jun. 2009.

[12] G. Georgoulas, C. Stylios, V. Kappatos, and E. Dermatas, "Wavelet usage for feature extraction for crack localization", IEEE 17th Mediterranen Conf. Control and Automation (MED), Greece pp. 15401545, June 2009.

[13] V. Kappatos, and E. Dermatas, "Neural localization of acoustic emission sources in ship hulls", J. Mar. Sci. Tech., vol. 14, pp. 248-255, 2009.

[14] G. Georgoulas, V. Kappatos, and G. Nikolakopoulos, "Acoustic emission localization on ship hull structures using a deep learning approach”, 23rd Int. Conf. Vibroengineering on Modeling, Identification and Fault Detection in Oil and Gas Equipment and Infrastructures, Turkey, vol. 9 pp. 56-61, 2016.

[15] K. R. Rao, and P. Yip, Discrete Cosine Transform: Algorithms, Advantages, Applications, CA: Academic Press Professional, 1990.

[16] J. G. Proakis, and D. G. Manolakis, Digital Signal Processing: Principles, Algorithms, and Applications. Prentice Hall, 1996.

[17] E. Keogh, K. Chakrabati, M. Pazzani, and S Mehtotra, "Dimensionality reduction for fast similarity search in large time series databases", Know. Inf. Syst., vol 3, pp. 263-286, Aug. 2001.

[18] K. Yi, and C. Faloutsos, "Fast time sequence indexing for arbitrary Lp norms," VLDB '00 Proceedings of the 26th Int. Conf. Very Large Databases, Egypt, pp. 297-306, Sept. 2000.

[19] P. Karvelis, G.Georgoulas, IP. Tsoumas, JA. Antonino-Daviu, V. Climente-Alarcon, and C. Stylios, "A Symbolic Representation Approach for the Diagnosis of Broken Rotor Bars in Induction Motors", IEEE Trans. Ind. Informat., vol. 11, pp. 1028-1037, Aug. 2015.

[20] SAX. Webpage: http://www.cs.ucr.edu/ eamonn/SAX.htm, Accessed 20 Sept. 2016.

[21] J. Schmidhuber, "Deep learning in neural networks: An overview", Neural Netw., vol 61, pp/ 85-117, Jan. 2015.

[22] VT. Tran, F. Althobiani, and A. Ball, “. An approach to fault diagnosis of reciprocating compressor valves using Teager-Kaiser energy operator and deep belief networks", Expert Syst. App., vol. 41, pp. 4113-4122, Jul. 2014.

[23] P. Tamilselvan, and P. Wang, "Failure diagnosis using deep belief learning based health state classification”, Reliab. Eng. Syst. Safe., vol. 115, pp. 124-135, Jul. 2013.

[24] Y. Bengio, P. Lamblin, D. Popovici, ND H. Larochelle, "Greedy layerwise training of deep networks", 19th Int. Conf. Neural Information Processing Systems (NIPS), Canada, vol 19, pp. 153-160, Dec. 2006.

[25] A. Nielsen, "Acoustic emission source based on pencil lead breaking", The Danish Welding Institute, Report 80-15, Denmark, 1980.

[26] IH. Witten, and E. Frank, Data Mining: Practical Machine Learning Tools and Techniques, CA: Morgan Kaufmann, 2005.

[27] M. Wasikowski, and X. Chen, "Combating the small sample class imbalance problem using feature selection", IEEE Trans. Knowl. Data Eng., vol 22, pp. 1388-1400, Oct. 2010.

[28] J. Heaton, Artificial Intelligence for Humans, Vol. 3: Deep Learning and Neural Networks, Create Space Independent Publishing Platform, Oct. 2015. 